\title{
Investigating Precision and Accuracy of a Robotic Inspection and Repair System
}

\author{
Miftahur Rahman, Haochen Liu*, Masoumeh Rahimi, Cristobal Ruiz Carcel, Leigh \\ Kirkwood, Isidro Durazo Cardenas, Andrew Starr* \\ School of Aerospace, Transport and Management, Cranfield University, Bedford MK43 OAL, United Kingdom
}

*E-mail address: haochen.liu@cranfield.ac.uk; a.starr@cranfield.ac.uk

\begin{abstract}
Robot integration in railway maintenance steps a prominent pavement in high-efficient and low-cost job execution for the infrastructure management. To achieve practical and diverse inspection and repair railway job, a robot manipulator on a locomotive platform is one of the best options. A lot of research has been conducted to find the accuracy and precision of industrial robotic manipulator where the manipulator base is fixed. This paper initiates an exploration of the accuracy and precision of a Robotic Inspection and Repair System (RIRS), which is a novel robotic railway maintenance system integrated with an industrial manipulator (UR10e) with 6 degree-of-freedom, mounting on an Unmanned Ground Vehicle (UGV) (Warthog) and specially designed trolley. In this research, a mimic track visual inspection test using QR code detection is adopted and implemented by an arm-mounted monocular camera. Then a sequential pose moves with multiple payload weights on the manipulator end has been performed as a performance measurement of repair jobs using a vision-based position tracking algorithm. The measurement results demonstrate that RIRS can maintain accurate and consistent performance in both defect position inspection and repair moves with diverse payloads. For inspection the positional error was only $0.27 \%$ while for repair moves the end-effector can reach the same position within $1 \mathrm{~mm}$. This research establishes a foundation for system command $\&$ control development and supporting more practical railway jobs deployment towards full autonomy for RIRS in the future.
\end{abstract}

Keywords: Railway Maintenance; Unmanned Ground Vehicle; Robotic automation system; Precision \& accuracy

\section{Introduction}

Railway is one of the safe and convenient mass transport systems for transporting people and goods. To keep pace with the increasing demand and ensuring a safe and comfortable journey, it is important to find the usage of modern robotics technology for rail inspection and repair. Using robotic system for rolling stock maintenance can provide financial advantages [1] or a specially designed train with sensors such as New Measurement Train (NMT) [2] can reduce the inspection time by a big margin. Though robotics and autonomous systems are used in railway sector for more than a decade, most of them are used in rolling stock maintenance and most of the system are without manipulation [3].

Wheeled robot or UGV like Warthog from Clearpath Robotics has capability to navigate in adverse terrain and can carry large amount of payload. Miniature wheeled robot for cleaning the track was proposed by James et. al. [4] but wheeled robot struggles to navigate along the track.. Combination of an UGV and a manipulator are called mobile manipulator. Mobile manipulators are capable of performing many dangerous and remote task such as tunnel inspection [5] or nuclear reactor cleaning [6]. For detecting defects in the track, an manipulator and wheeled robot combination was proposed by [7] but it's navigation capability is only limited to track. While Railpod [8] can navigate both on-track and off-track with its reconfigurable wheel mechanism but lacks a manipulator for repair or close inspection. To remove these barriers and to find a versatile solution for railway track inspection and repair work, an autonomous RIRS has been proposed in [9] where a trolley has been added to extend the navigation capability of mobile manipulator for on-track operation.

Industrial manipulator is a major component of the proposed RIRS and the performance of RIRS for the inspection and repair job partly depends on the accuracy and precision of the industrial manipulator. Industrial manipulators are used in many complex tasks such as painting, pick and place, cut, assembly and etc.[10]. However, manipulators motion control was stationary in these tasks. In [11], Huang et. al. proposed a method of selecting a manipulator by calculating the reliability index based on positional accuracy. While Dandash et. al. proposed a method to find the repeatability of an industrial manipulator by measuring the orientation of each joints considering the payload and different points in workspace [12]. After analysing the repeatability 
with respect to various load for an industrial manipulator, Brethe et. al. concluded that higher load deteriorates pose accuracy and repeatability [13]. Therefore, to achieve RIRS railway maintenance, the hardware motion positional performance testing is necessary.

Railway maintenance demands high accuracy and precision and considers the human safety and comfort at the same time. For example, tolerance set by Network Rail for recording the track geometry of top side should be below $1 \mathrm{~mm}$ [14]. Not only the robot manipulator but also the whole RIRS system should be investigated for positional performance since the scale of RIRS demonstrator dimension and $\mathrm{UGV}$ motion is significantly less than the railway maintenance. During inspection, if defect positioning accuracy is not satisfactory then the defect position will be registered in wrong place while positional inaccuracy and less precision will lead the RIRS to perform repair job in the wrong place. Moreover, the manipulator should be able to achieve various positioning moves in the workspace with carrying different payload necessities. This research initiates the investigation and analysis of the accuracy and precision of RIRS for basic inspection and repair works.

In the second section, the specification of a RIRS system and functions of major components such trolley, UGV and manipulator will be introduced. Definitions of accuracy and precision for a RIRS and methodology of this research will be described in the third section. Furthermore, the measurement strategy by object positioning using monocular camera will be discussed briefly. In the fourth section, design of experiments and the performance results will be discussed, and data will be analysed. At last, the conclusion and impact are stated in fifth section.

\section{RIRS}

RIRS is an autonomous robotic system that is a combination of a Warthog UGV, UR10e manipulator and a trolley. The proposed RIRS is shown in Fig 1. Key specifications of major components are given in Table 1 .

Table 1. Key specifications of various RIRS components

\begin{tabular}{|c|l|}
\hline \multirow{3}{*}{ Warthog } & $\begin{array}{l}\text { OEM: Clearpath Robotics } \\
\text { Wheel number: } 4,20 \text { " AGV tyre } \\
\text { Vehicle Dimension: } 1.52 \times 1.38 \text { x } 0.83 \mathrm{~m} \\
\text { Drive type: Differential drive } \\
\text { Payload capacity: Maximum } 272 \mathrm{~kg}\end{array}$ \\
\hline \multirow{3}{*}{ ORM: Clearpath Robotics } \\
& $\begin{array}{l}\text { Number of Joints: 06 } \\
\text { Payload capacity: Maximum } 10 \mathrm{~kg} \\
\text { Maximum Reach: } 1300 \mathrm{~mm}\end{array}$ \\
\hline Wrist Camera & OEM: Robotiq Inc \\
\hline
\end{tabular}

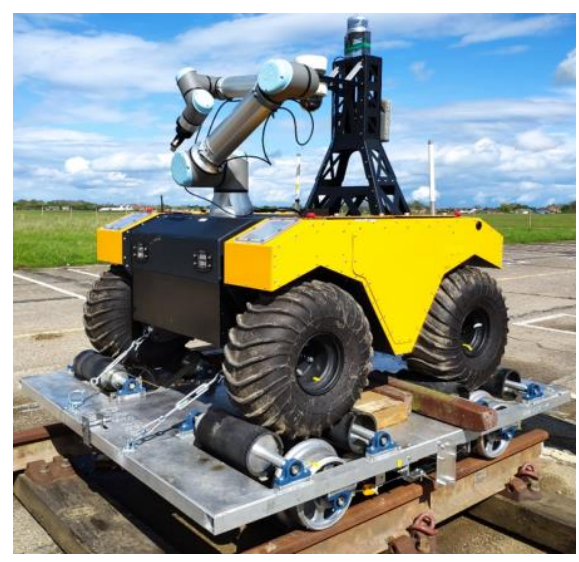

Fig. 1. Proposed RIRS on the track

\begin{tabular}{|c|l|}
\hline \multirow{3}{*}{ Trolley } & $\begin{array}{l}\text { Focus length: 30mm to infinity } \\
\text { Resolution: Maximum 5 megapixel } \\
\text { Autofocus: Yes }\end{array}$ \\
\hline \multirow{3}{*}{ 2-finger Gripper } & $\begin{array}{l}\text { OEM: Robotiq Inc } \\
\text { Stroke length: } 85 \mathrm{~mm}\end{array}$ \\
& $\begin{array}{l}\text { OEM: custom design and made. } \\
\text { Dimension: } 2.0 \times 1.78 \mathrm{~m} \\
\text { Wheel Number: } 04,10 \text { " rail wheel } \\
\text { Number of Contact Roller: 08 } \\
\text { Power transmission: belt and pulley }\end{array}$ \\
\hline
\end{tabular}

RIRS is equipped with an onboard IMU sensor, and all the wheels have a wheel encoder which can provide odometry information. For processing all the sensor data, it has an onboard computer. It also carries a sensor tower which carries a rotatable camera for environment monitoring, RTK-GPS module for positioning and VLP-16 Lidar for 3D mapping. It comes with ROS preinstalled.

The advantage of attaching the wrist camera to a manipulator instead of a fixed position is that camera can be moved to different positions for a closer look and inspecting the objects from different views.

Because of the pneumatic wheels, selected Warthog UGV do not have the capability to run along the railway track. A specially designed trolley can carry Warthog and ensure navigation along the track. At first, power is transmitted from the Warthog to contact rollers and then the power is transferred to a 10-inch wheel. Fig 2 shows a fabricated trolley for RIRS.

\section{Methodology}

Accuracy and precision (also considered as repeatability) are important for a robotic system to measure performance and effectiveness. For positioning measurement, accuracy means how close the result is related to a reference, while precision refers to the consistency of the results of multiple repetitive motion. For a mobile manipulator, 


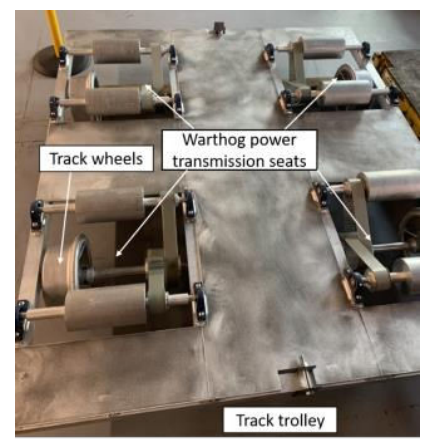

Fig. 2. Components of specially designed trolley for RIRS

accuracy and precision can be measured from the pose (position and orientation) of end-effector in a workspace like in Fig 3 [15].

For RIRS performance measurement in this research, the inspection accuracy is measured with the detected defect position referencing to the initial RIRS position. The inspection precision can be evaluated by comparing the detected defect position between multiple times for inspection execution. Finally, the repair precision is measured by arm reaching the same end-effector pose for multiple times.

The detected defect position from initial RIRS position is shown in Fig 4. The detected defect position to the initial RIRS position of can be found by following Eq. (1):

$$
d=a+b+c
$$

Where $a, b, c, d$ denotes the position information by following:

d: defect position from initial RIRS position a: distance from initial RIRS position to final RIRS position

$b$ : distance from manipulator base to manipulator tip

c: distance from camera frame to defect

Defect position detected by RIRS depend on three measurements: mobile base (Warthog and trolley) movement from initial RIRS position to final position, end-effector position from mobile base to camera frame, and defect position from camera to the defect center.

Distance traveled by the RIRS, $a$, can be found from the odometry sensing, from the LiDAR or

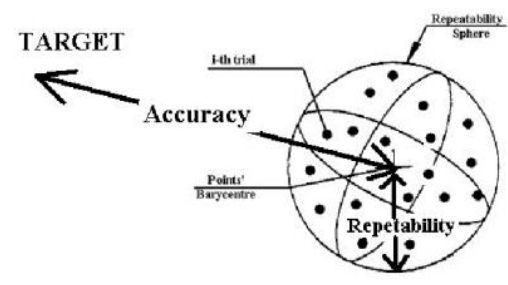

Fig. 3. Accuracy and precision of a manipulator

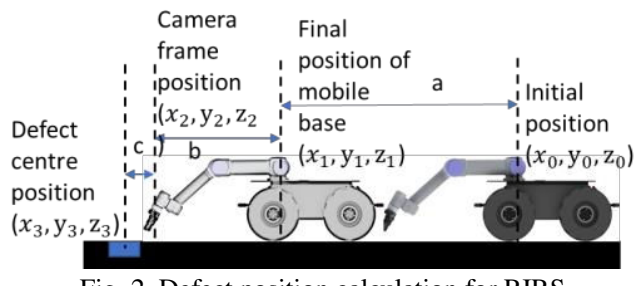

Fig. 2. Defect position calculation for RIRS

using any distance measuring technique. Forward kinematics can be used for finding the end-effector position from RIRS base, $b$ [16], [17]. End-effector position can be calculated from the known values of joint angles and length. UR10e manipulator has 6 link and final transformation of the end-effector can be found by multiplying the transformation of each link.

$$
o_{T_{6}=} o_{T_{1}} \cdot 1_{T_{2}} \cdot 2_{T_{3}} \cdot 3_{T_{4}} \cdot 4_{T_{5}} \cdot 5_{T_{6}}
$$

The transformation between two consecutive links can be found using following Eq. (3) where one frame can be transformed into another frame using rotation and translation ( $c$ denotes $\cos$ and $s$ denotes $\sin )$ :

$$
{ }^{i-1} T_{i}=\left[\begin{array}{cccc}
c \theta_{i} & -s \theta_{i} & 0 & a_{i-1} \\
s \theta_{i} c \alpha_{i-1} & c \theta_{i} c \alpha_{i-1} & s \alpha_{i-1} & s \alpha_{i-1} d_{i} \\
s \theta_{i} s \alpha_{i-1} & c \theta_{i} s \alpha_{i-1} & c \alpha_{i-1} & c \alpha_{i-1} d_{i} \\
0 & 0 & 0 & 1
\end{array}\right] \text { (3) }
$$

Fortunately, Robot Operating Software (ROS) provides package named, tf, for calculating the transformation between the frames of a robot [18]. The advantage of $\mathrm{tf}$ is that it can keep tracking of dynamic changes between multiple frames. Using the following equation $\mathrm{tf}$ in ROS extends the transformation frame not only in space but also in time.

$$
T_{a @ t_{0}}^{c @ t_{1}}=T_{a @ t_{0}}^{b @ t_{0}} * T_{b @ t_{1}}^{b @ t_{0}} * T_{b @ t_{1}}^{c @ t_{1}}
$$

where $T_{a @ t_{0}}^{c @ t_{1}}$ denotes transformation between frame $c$ to $a$ in two different time $t_{0}$ and $t_{1}$. This transformation is achieved by 3 transformations: the first one is calculating transformation from frame $b$ to $a$ in time $t_{0}$, then transformation of frame $b$ in two different timestamps and finally, finding the transformation of frame $c$ with respect to frame $b$ in time $t_{1}$. The transformation between frames using ROS tf is shown in Fig. 5. Lastly, the position of

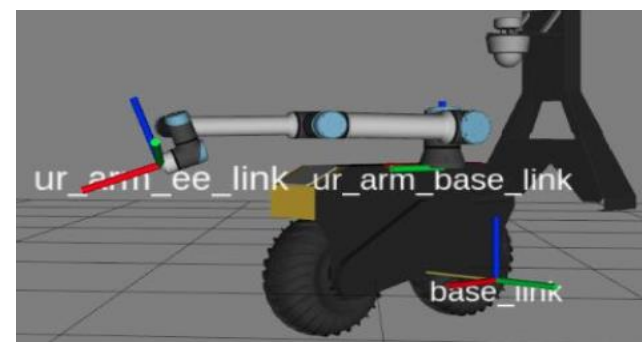

Fig. 3. Transformation between two coordinate frames 
defect from the camera frame can be found by using ViSP (Visual Servoing Platform). ViSP auto tracker is an open-source real-time 3D model-based tracker built on the principle of ViSP. For finding the transformation of a feature point in the object with respect to the camera frame, ViSP auto tracker uses points transfer method [19]. If a point is denoted in frame 1 as ${ }^{1} P$, then the coordinates of the same point in frame 2 can be found using the following equation:

$$
{ }^{1} p={ }_{1}^{2} \operatorname{tr}\left({ }^{1} p\right)=\alpha_{1}^{2} H^{1} p
$$

Here, ${ }_{1}^{2} H$ is the homography which defines transformation between two frames at a scale factor $\alpha$.

The inspection accuracy of the RIRS system is measured by the whole distance from the defect center position $\left(x_{3}, y_{3}, z_{3}\right)$ to the initial RIRS position $\left(x_{0}, y_{0}, z_{0}\right)$. The inspection precision is measured by the consistency of multiple repetitions.

For the repair performance, the precision is measured by multiple repetitions of a same repair pose motions, which are executed with and without carrying different payload of weights.

\section{Experiments and Results 4.1. Inspection}

As a dummy defect, a QR code has been attached on top of left rail in Fig. 6(a). An initial RIRS position was marked, and the manipulator was set in an inspection pose so that the monocular camera can see the left rail. The inspection process was started by running a python code and ViSP auto tracker simultaneously. Programmed code moves the RIRS forward with constant speed and detects QRcode position by ViSP auto tracker algorithm. ViSP auto tracker can stably extract and publish the position and orientation, specifically the relative center position of QRcode to the camera. Once QRcode is detected, the RIRS stops and keep detecting, and it will create a bounding box and display the axis similar to Fig. 6(b). RIRS was again moved back to the marked initial position and rerun total of 10 times for the same QRcode position.

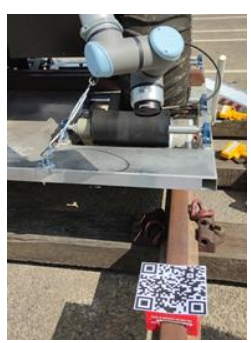

(a)

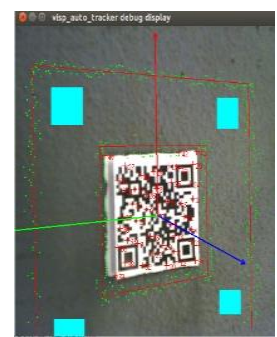

(b)
Fig. 4. QRcode for inspection task, (a) QRcode on the track (b) ViSp_auto_tracker window showing bounding box and axis after detection.
To find the defect position from the initial RIRS position, 3 distances were recorded and summed together. The distance travelled by RIRS was recorded by a laser sensor, distance from manipulator base to manipulator tip was calculated using ROS tf and defect position from camera frame was found from ViSP auto tracker. Then these three distances were computed to find the defect position from initial RIRS position. The real defect position is also captured as a reference by a laser point measure kit. The measured defect position and real defect position were compared to find out the accuracy of system. The error in every instance with respect to the mean was calculated to find the precision. Fig 7(a) and 7(b) shows the measured result for error in defect position to find the performance of RIRS in inspection.

The maximum error in finding defect position is $56 \mathrm{~mm}$ while smallest error is $17 \mathrm{~mm}$ and the error range is $38.72 \mathrm{~mm}$. The actual defect position from the initial RIRS position is $3.75 \mathrm{~m}$ and the average inspection error in defect position is $0.27 \%$.

\subsection{Repair motion}

Once the inspection process was finished, two different poses with varying angle were defined for the manipulator; one is straight pose, and another is repair pose. Also, payloads were attached to the manipulator after each set of experiments. Every time manipulator will perform motion from straight pose to repair pose with carrying the payload while UGV was stationary and QRcode position was fixed. Three different payload was created with
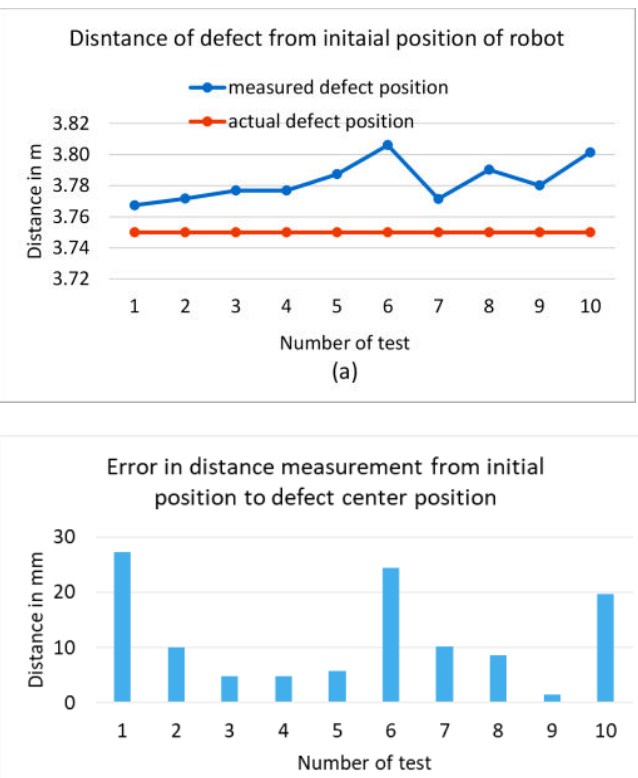

(b)

Fig. 5. Error investigation for inspection job of RIRS. (a) mean and actual defect position from initial RIRS position (b) error in every instance 


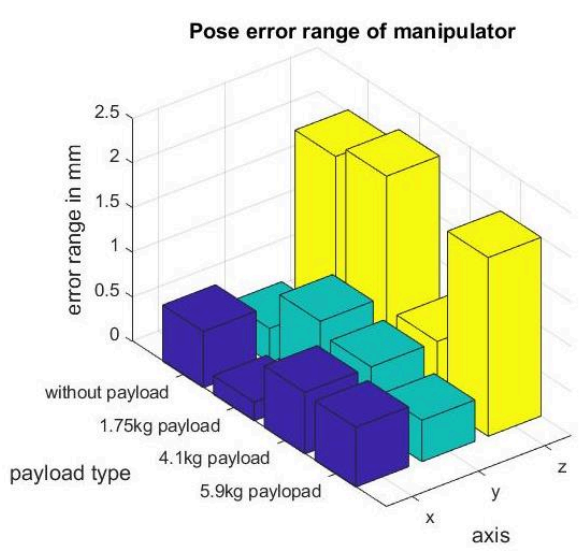

Fig. 8: Difference between maximum and minimum position in $3 \mathrm{D}$ coordinate

combination of 1 rail clip and 1 nut-bolt $(1.75 \mathrm{~kg}), 1$ rail clip and 3 nut-bolt $(4.1 \mathrm{~kg})$ and 3 rail clip and 3 nut-bolt $(5.9 \mathrm{~kg})$. ROS Moveit was used to control the manipulation and ViSP was used to record the relative distance from camera to QRcode. For every payload, the test was conducted 10 times to find the average.

Maximum and minimum of positions in every axis was calculated and then the range was determined, which is shown in Fig. 8. The range for deviation was bigger in $\mathrm{z}$ axis than the other axis.

In Fig. 9(a), range for manipulator position without payload in $3 \mathrm{D}$ space is $[0.63 \mathrm{~mm}, 0.38 \mathrm{~mm}$ and $2.00 \mathrm{~mm}$ ]. Fig. 9(b). shows recorded pose of UR10e manipulator after 10 repair motions with $1.75 \mathrm{~kg}$ payload from initial pose. Few poses deviate more the mean and deviation in $3 \mathrm{D}$ space is [0.21mm, $0.83 \mathrm{~mm}, 2.17 \mathrm{~mm}]$. End-effector pose with $4.1 \mathrm{~kg}$ payload after 10 repair motion in shown in Fig. 9(c). 3 points are far from mean and error range increases both in $\mathrm{x}$ and $\mathrm{y}$ axis compared to no payload condition to $0.69 \mathrm{~mm}$ and $0.438 \mathrm{~mm}$, respectively. Fig. 9(d) shows the end-effector pose with $5.9 \mathrm{~kg}$ payload after 10 repair motion from initial pose. Points are much scattered, and the range of deviation is biggest in all 3 axes. The measured precision deviation range is $[0.67 \mathrm{~mm}, 0.46 \mathrm{~mm}$, $2.00 \mathrm{~mm}]$ in $3 \mathrm{D}$ space.

\subsection{Result analysis}

The designed tests effectively measure the 3D positional performance of manipulator for inspection and repair moves.

During the inspection, the whole RIRS system is moving, and positional error was $32.96 \mathrm{~mm}$ or $0.27 \%$ to the whole distance $3.76 \mathrm{~m}$. The main reasons for this positional inaccuracy were the slippage between warthog wheel and contact rollers of the trolley, initial RIRS position mismatch etc. A small percentage of error demonstrate the capability of

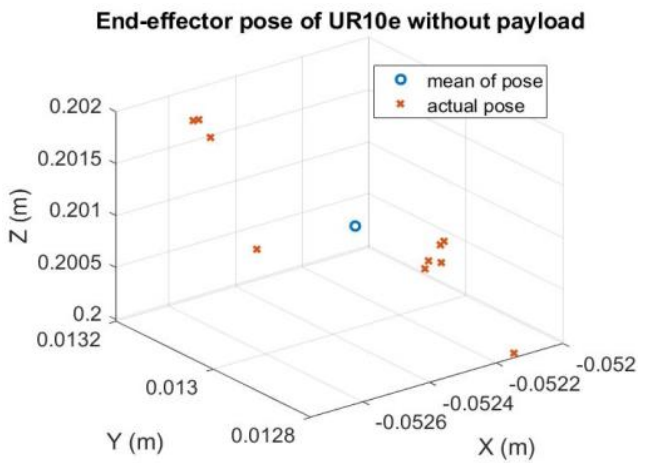

(a)

End-effector pose of UR10e with $1.75 \mathrm{~kg}$ payload

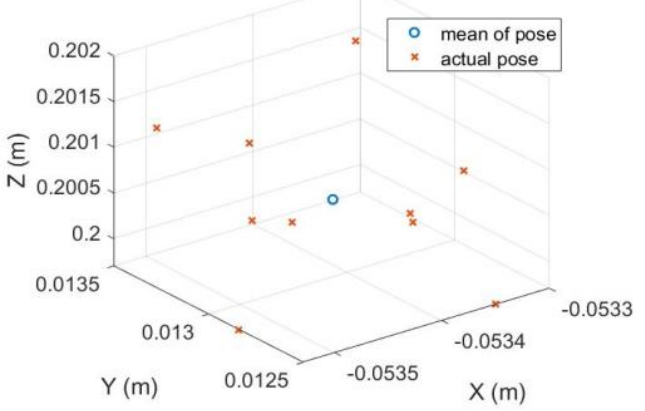

(b)

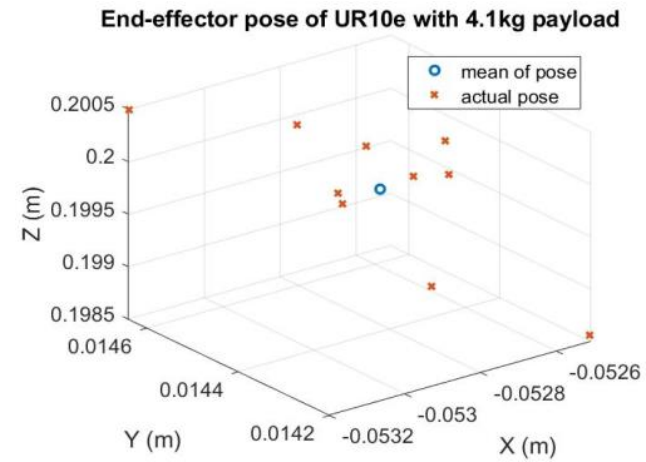

(c)

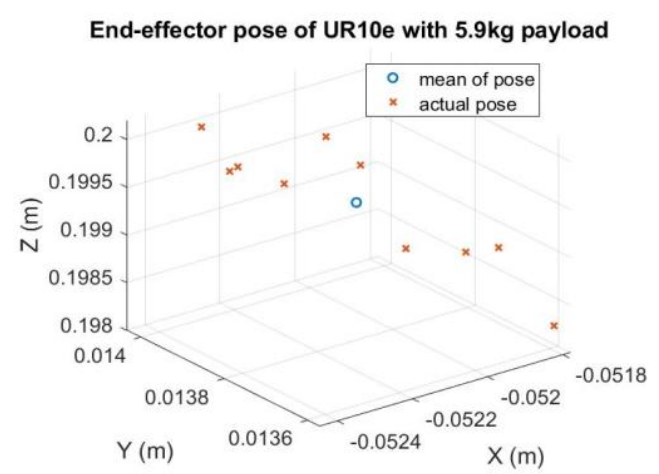

(d)

Fig. 9. Pose error for UR10e manipulator pose without payload from 10 repair motion, (a) without payload (b) with $1.75 \mathrm{~kg}$ payload, (c) with $4.1 \mathrm{~kg}$ payload, (d) with $5.9 \mathrm{~kg}$ payload.

RIRS system for rail inspection job. Accuracy can be improved by incorporating the slippage amount in the program and setting the RIRS initial position more precisely. 
During repair, the manipulator was able to reach the position with very high accuracy, less than $1 \mathrm{~mm}$ deviation from the mean position and around $2 \mathrm{~mm}$ in $\mathrm{z}$ axis for every repair motion. A bigger error in $\mathrm{z}$ axis was expected as only monocular camera was used which is not good for finding distance in $\mathrm{z}$ direction. High precision proves the feasibility of the manipulator for RIRS. This small deviation may cause by the vibration of robot base during arm manipulation and ViSP auto tracker object position calculation. For finding the end-effector position more accurately, laser-based sensors can be used instead of VISP auto tracker and robot base vibration can be reduced by improving the path planning and speed of manipulator.

\section{Conclusion}

In this research, the positional accuracy and precision of the proposed RIRS system have been investigated for railway track inspection and repair job. A robotic UGV with an industrial robot manipulator is applied as a maintenance job testing demonstrator. A basic on-track defect position inspection test is conducted to measure the positional accuracy and precision of the whole RIRS system by integration of vehicle motion, system structural dimension and relative distancing from defect to inspection tool. The results demonstrate that the RIRS demonstrator can maintain good accuracy and precision for basic inspection job. Additional tests consisting of multiple payload weights on the manipulator end were implemented to measure the precision of repair moves. The robot manipulator was able to reach the same position with high precision with different payloads. It indicates the feasibility of the integrated UR10e manipulator for accurate railway maintenance jobs. This research provides effective performance measurement for the RIRS demonstrator and on-board robot manipulator, establishing a foundation for command \& control development, and supporting more practical railway jobs deployment for the RIRS towards full autonomy in the future.

\section{Acknowledgements}

This work was supported by the Shift2Rail Joint Undertaking under the European Union's Horizon 2020 research and innovation programme (Grant agreement IN2SMART2, No. 881574 and IN2TRACK2, No. 826255).

\section{References}

[1] R. K. W. Vithanage, C. S. Harrison, and A. K. M. M. DeSilva. Enhance 3D Point Cloud Accuracy Through Supervised Machine Learning for Automated Rolling Stock
Maintenance: A Railway Sector Case Study. in 2018 International Conference on Computing, Electronics Communications Engineering (iCCECE), Aug. 2018, p. 242246.

[2] New Measurement Train (NMT) - Network Rail. https://www.networkrail.co.uk/running-the-railway/lookingafter-the-railway/our-fleet-machines-and-vehicles/newmeasurement-train-nmt

[3] R. Vithanage, C. Harrison, and A. DeSilva. Importance and Applications of Robotic and Autonomous Systems (RAS) in Railway Maintenance Sector: A Review, Computers; 2019. vol 8, p. 56.

[4] J. James, J. Wilson, J. Jetto, A. Thomas, and V. K. Dhahabiya. Intelligent track cleaning robot. in 2016 IEEE International Conference on Mechatronics and Automation; Aug. 2016. p. 332-337.

[5] E. Menendez, J. G. Victores, R. Montero, S. Martínez, and C. Balaguer. Tunnel structural inspection and assessment using an autonomous robotic system. Automation in Construction; Mar. 2018. vol. 87, p. 117-126.

[6] A. Kitamura, T. Namekawa, K. Hiramatsu, and Y. Sankai. Operating Manipulator Arm by Robot Suit HAL for Remote In-Cell Equipment Maintenance. Nuclear Technology; Dec. 2013. vol. 184, no. 3, p. 310-319.

[7] H. Rowshandel.The development of an autonomous robotic inspection system to detect and characterise rolling contact fatigue cracks in railway track. p. 195.

[8] Our Platform - RailPod Inc.

[9] M. Rahman, H. Liu, I. D. Cardenas, A. Starr, A. Hall, and R. Anderson. Towards an Autonomous RIRS: Design, Structure Investigation and Framework. in 2021 7th International Conference on Mechatronics and Robotics Engineering (ICMRE); Feb. 2021. p. 164-168.

[10] Y. Huang, Y. S. Yong, R. Chiba, T. Arai, T. Ueyama, and J. Ota. Kinematic Control With Singularity Avoidance for Teaching-Playback Robot Manipulator System. IEEE Transactions on Automation Science and Engineering; Apr. 2016. vol. 13, no. 2, p. 729-742.

[11] Y. Huang et al.. Evaluation of Motion Reliability for Robot Manipulator. in 2018 International Conference on Manipulation, Automation and Robotics at Small Scales (MARSS); Jul. 2018, p. 1-5.

[12] D. Dandash, J.-F. Brethé, and E. Vasselin, Modeling of the orientation repeatability for industrial manipulators; 2010. p. 2975.

[13] J.-F. Brethé, E. Vasselin, D. Lefebvre, and B. Dakyo. Modelling of repeatability phenomena using the stochastic ellipsoid approach. Robotica; Jul. 2006. vol. 24, no. 4, p. 477490.

[14] Network Rail, Track Geometry Recording;1995.

[15] A. Hijazi, D. Lefebvre, and J. Brethé. Characterization of repeatability of XY-Theta platform held by robotic manipulator arms using a camera. 11th International Conference on Informatics in Control, Automation and Robotics; 2014.

[16] R. P. Paul, Robot Manipulators: Mathematics, Programming, and Control : the Computer Control of Robot Manipulators. Richard Paul; 1981.

[17] A. Solyman. Computing the Forward Kinematics of 6DOF Robotic Arm; Jan. 2019.

[18] T. Foote. tf: The transform library. in 2013 IEEE Conference on Technologies for Practical Robot Applications (TePRA), Woburn, MA, USA, Apr. 2013, p. 1-6.

[19] A. I. Comport, E. Marchand, M. Pressigout, and F. Chaumette. Real-time markerless tracking for augmented reality: the virtual visual servoing framework. IEEE Transactions on Visualization and Computer Graphics, Jul. 2006. vol. 12, no. 4, p. 615-628. 
$2021-10-20$

\section{Investigating precision and accuracy of a robotic inspection and repair system}

Rahman, Miftahur

SSRN

Rahman M, Liu H, Rahimi M, et al., (2021) Investigating precision and accuracy of a robotic inspection and repair system. In: 10th International Conference on Through-life Engineering Services (TESConf 2021), 16-17 November 2021, Twente, The Netherlands https://dspace.lib.cranfield.ac.uk/handle/1826/17300 Downloaded from Cranfield Library Services E-Repository 